\title{
A ferroelectric quantum phase transition inside the superconducting dome of $\mathrm{Sr}_{1-x} \mathrm{Ca}_{x} \mathrm{TiO}_{3-\delta}$
}

\author{
Carl Willem Rischau1, Xiao Lin ${ }^{1,2}$, Christoph P. Grams ${ }^{2}$, Dennis Finck ${ }^{2}$, Steffen Harms ${ }^{2}$, \\ Johannes Engelmayer ${ }^{2}$, Thomas Lorenz ${ }^{2}$, Yann Gallais ${ }^{3}$, Benoît Fauqué ${ }^{1,4}$, Joachim Hemberger ${ }^{2}$ \\ and Kamran Behnia ${ }^{1 \star}$
}

$\mathrm{SrTiO}_{3}$, a quantum paraelectric ${ }^{1}$, becomes a metal with a superconducting instability after removal of an extremely small number of oxygen atoms ${ }^{2}$. It turns into a ferroelectric upon substitution of a tiny fraction of strontium atoms with calcium ${ }^{3}$. The two orders may be accidental neighbours or intimately connected, as in the picture of quantum critical ferroelectricity ${ }^{4}$. Here, we show that in $\mathrm{Sr}_{1-x} \mathrm{Ca}_{x} \mathrm{TiO}_{3-\delta}(0.002<x<0.009$, $\delta<0.001$ ) the ferroelectric order coexists with dilute metallicity and its superconducting instability in a finite window of doping. At a critical carrier density, which scales with the $\mathrm{Ca}$ content, a quantum phase transition destroys the ferroelectric order. We detect an upturn in the normal-state scattering and a significant modification of the superconducting dome in the vicinity of this quantum phase transition. The enhancement of the superconducting transition temperature with calcium substitution documents the role played by ferroelectric vicinity in the precocious emergence of superconductivity in this system, restricting possible theoretical scenarios for pairing.

A perovskite of the $\mathrm{ABO}_{3}$ family, $\mathrm{SrTiO}_{3}$ is a quantum paraelectric whose dielectric constant rises to $\sim 20,000$ at low temperature ${ }^{1}$, but avoids long-range ferroelectric order. It becomes a metal by substituting $\mathrm{Sr}$ with $\mathrm{La}, \mathrm{Ti}$ with $\mathrm{Nb}$, or by removing $\mathrm{O}$. It has been known for half a century that this metal is a superconductor at low temperatures ${ }^{2}$. More recently, a sharp Fermi surface and a superconducting ground state have been found to persist down to a carrier concentration of $10^{17} \mathrm{~cm}^{-3}$ in $\mathrm{SrTiO}_{3-\delta}$ (refs 5,6). In this range of carrier concentration, exceptionally low compared to any other known superconductor, the Fermi temperature is an order of magnitude lower than the Debye temperature and the ability of phonons to form Cooper pairs is questionable ${ }^{7,8}$. Recent theories invoke fluctuations of the ferroelectric mode ${ }^{9}$, longitudinal optical phonons ${ }^{10}$ or plasmons ${ }^{8}$ as bosons responsible for the generation of Cooper pairs.

In many unconventional superconductors, another electronic order competes with superconductivity. Here, the ferroelectric order is an obvious candidate ${ }^{4,9}$. It emerges in $\mathrm{SrTiO}_{3}$ by isotopic substitution of ${ }^{16} \mathrm{O}$ oxygen atoms with ${ }^{18} \mathrm{O}$ (ref. 11), application of stress ${ }^{12}$ or substitution of a tiny fraction of Sr with Ca (ref. 3). However, mobile electrons screen polarization and therefore only insulating solids are expected to host a ferroelectric order. Hitherto, as a paradigm, ferroelectric quantum criticality, in contrast to its magnetic counterpart, was deprived of an experimental phase diagram in which a superconducting phase and a ferroelectric order share a common boundary.
Here, we produce such a phase diagram in the case of $\mathrm{Sr}_{1-x} \mathrm{Ca}_{x} \mathrm{TiO}_{3-\delta}$. The main new observations are the following: metallic $\mathrm{Sr}_{1-x} \mathrm{Ca}_{x} \mathrm{TiO}_{3-\delta}$ hosts a phase transition structurally indistinguishable from the ferroelectric phase transition in insulating $\mathrm{Sr}_{1-x} \mathrm{Ca}_{x} \mathrm{TiO}_{3}$; the coexistence between this ferroelectric-like order and superconductivity ends beyond a threshold carrier concentration; and, in the vicinity of this quantum phase transition, calcium substitution enhances the superconducting critical temperature and induces an upturn in the normal-state resistivity.

Figure 1 summarizes what we know about the emergence of ferroelectricity, metallicity and superconductivity in this system. When a small fraction of $\mathrm{Sr}$ atoms $(x>0.002)$ is replaced with isovalent $\mathrm{Ca}, \mathrm{Sr}_{1-x} \mathrm{Ca}_{x} \mathrm{TiO}_{3}$ becomes ferroelectric ${ }^{3}$, with a Curie temperature steadily increasing with $\mathrm{Ca}$ content in the dilute limit $0.002<$ $x<0.02$ (refs 3,13,14). Macroscopic polarization below the Curie temperature has been observed in dielectric and linear birefringence measurements, and found to build up in the plane perpendicular to the tetragonal axis along the $[110]$ and $[1 \overline{10}]$ directions ${ }^{3,14}$. Figure $1 \mathrm{~b}$ presents the temperature dependence of the real part of the dielectric permittivity $\varepsilon^{\prime}$ in our $\mathrm{Sr}_{1-x} \mathrm{Ca}_{x} \mathrm{TiO}_{3}$ single crystals at three different Ca contents $(x=0.0022 ; 0.0045 ; 0.009)$, obtained by measuring their complex conductivity. In the Ca-substituted system, there is a peak in the low-temperature permittivity at the Curie temperature, $T_{\mathrm{C}}$, and below $T_{\mathrm{C}}$, the polarization $P$ shows a hysteresis loop.

In $\mathrm{SrTiO}_{3}$, electric permittivity increases steadily with decreasing temperature and saturates at low temperatures due to quantum fluctuations, attaining a magnitude as large as to $20,000 \varepsilon_{0}$ (refs 1 , $15)$. The ionic radius of $\mathrm{Ca}(0.99 \AA)$ is smaller than $\mathrm{Sr}(1.12 \AA)$, and $\mathrm{CaTiO}_{3}$ loses its cubic symmetry at $1,600 \mathrm{~K}$ and suffers multiple structural transitions ${ }^{16}$ without becoming ferroelectric. The stabilization of the ferroelectric order in dilute $\mathrm{Sr}_{1-x} \mathrm{Ca}_{x} \mathrm{TiO}_{3}$ is restricted to a narrow window in the dilute limit of $\mathrm{Ca}$ content $(0.002<x<0.02)$ within a highly polarizable matrix. There are two alternative ways to picture this order. In the first one, it is driven by dipole-dipole interaction between off-centre Ca atoms, which form polarized clusters growing in size and percolating at the Curie temperature. In the second picture, the ferroelectric order is stabilized because the quantum fluctuations of the host matrix are pinned by Ca sites. A transverse Ising model with appropriate parameters $^{17}$ can reproduce the critical doping for the emergence of ferroelectricity as well as the subsequent increase in the Curie temperature with $x$ (ref. 3 ).

By heating $\mathrm{SrTiO}_{3}$ in vacuum, oxygen atoms are removed and mobile carriers are introduced ${ }^{18}$. A metal-insulator transition

\footnotetext{
${ }^{1}$ Laboratoire Physique et Etude de Matériaux (UMR 8213 CNRS-ESPCI), PSL Research University, 75005 Paris, France. ${ }^{2}$ II. Physikalisches Institut, Universität zu Köln, 50937 Köln, Germany. ${ }^{3}$ Laboratoire Matériaux et Phénomènes Quantiques (UMR 7162 CNRS-Paris Diderot), 75013 Paris, France. ${ }^{4}$ JEIP, USR 3573 CNRS, Collège de France, 75005 Paris, France. *e-mail: kamran.behnia@espci.fr
} 
a

$$
\mathrm{Sr}_{1-x} \mathrm{Ca}_{x} \mathrm{TiO}_{3}(0.002<x<0.02)
$$

Ferroelectric
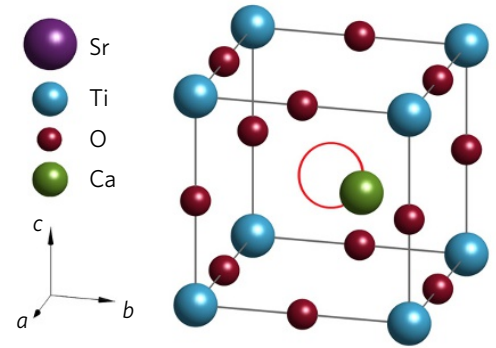

b

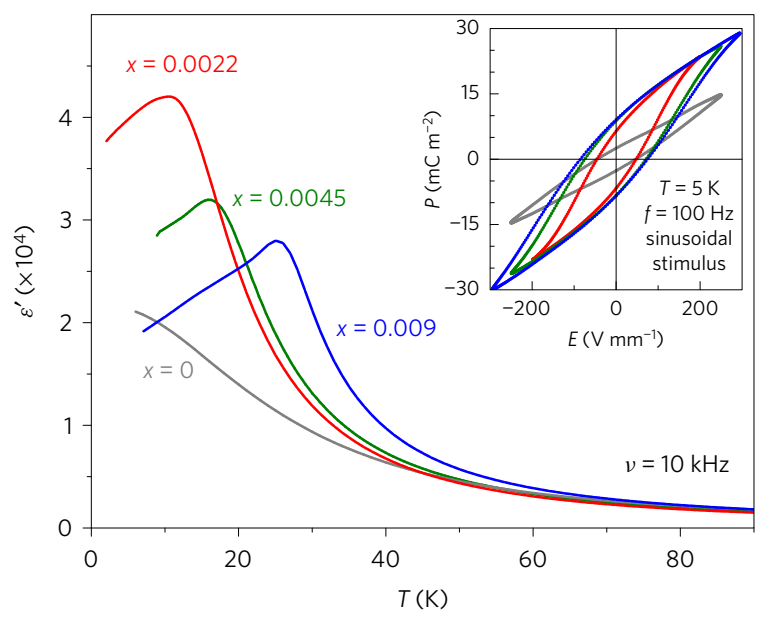

c

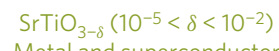

Metal and superconductor

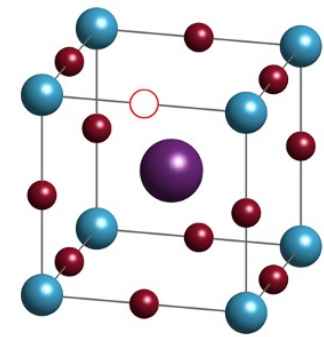

d
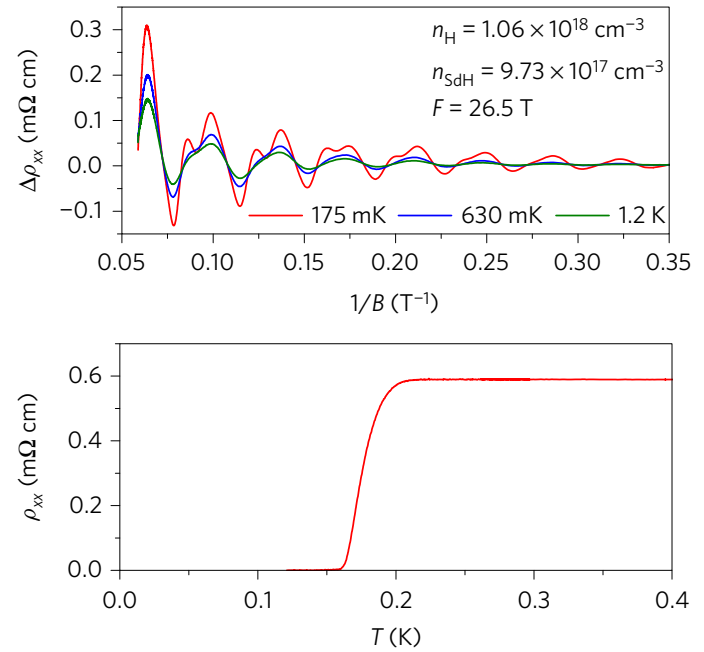

Figure 1 | Emergence of ferroelectricity and metallicity by atomic substitution in $\mathrm{SrTiO}_{3}$. a, Substituting strontium with isovalent and smaller calcium atoms leads to ferroelectricity. Smaller calcium atoms can take off-centre positions and create local electric dipoles. Above a critical Ca threshold, a long-range ferroelectric order emerges below a Curie temperature. $\mathbf{b}$, Ferroelectricity in insulating $\mathrm{Sr}_{1-x} \mathrm{Ca}_{x} \mathrm{TiO}_{3}$ documented by the temperature dependence of the real component of the dielectric permittivity, $\varepsilon^{\prime}$, for three different $x$. The maximum in $\varepsilon^{\prime}$ marks the Curie temperature. The inset shows polarization-electric field hysteresis loops at $T=5 \mathrm{~K}$. c, Removing an oxygen atom introduces two $\mathrm{n}$-type carriers. d, Dilute metallic $\mathrm{SrTiO}_{3}$ displays quantum oscillations of resistivity and a superconducting transition. The size of the Fermi surface according to the frequency of quantum oscillations (with period $F$ ) matches the carrier density $(n)$ given by the Hall coefficient ${ }^{5,6}$.

is expected above a threshold carrier concentration ${ }^{19}$, which is exceptionally low because of the long Bohr radius ${ }^{5}$. At a carrier density of $10^{17} \mathrm{~cm}^{-3}$, several orders of magnitude above the expected threshold of metal-insulator transition, there is a single Fermi $\mathrm{sea}^{20}$ (and not a collection of metallic puddles). This picture is based on the observation of quantum oscillations (Fig. 1d), with frequencies ${ }^{5,6,21}$ matching the carrier density expected from the magnitude of the Hall coefficient. This dilute metal is subject to a superconducting instability ${ }^{2,5,6}$. The aim of this study is to find what happens to metallicity and ferroelectricity when one removes oxygen and substitutes Sr with Ca.

The first new result of this study is presented in Fig. 2, which shows the persistence of a phase transition structurally identical to the ferroelectric phase transition in dilute metallic $\mathrm{Sr}_{1-x} \mathrm{Ca}_{x} \mathrm{TiO}_{3-\delta}$. As seen in Fig. 2a, the electric resistivity of these samples shows an anomaly at the Curie temperature of the parent insulating sample (see Fig. 1b). This observation implies that even in the presence of mobile electrons, the system goes through a ferroelectric-like phase transition at the same temperature. We checked the presence of this phase transition by two thermodynamic probes. Thermal expansion data are presented in Fig. $2 b$. There is a clear anomaly at the Curie temperature. Its magnitude and the temperature at which it occurs are identical in an insulating and a dilute metallic sample with the same calcium content. Our data on sound velocity (see Supplementary Information) confirm this. We can therefore safely conclude that the phase transition giving rise to an anomaly in the resistivity of metallic samples (when the carrier concentration is in the range of $10^{17} \mathrm{~cm}^{-3}$ ) is structurally identical to the one causing the peak in permittivity in the insulating sample. Further evidence is provided by Raman spectroscopy (Fig. 2c,d). The entrance to the ferroelectric state is concomitant with the activation of two transverse optical (TO) phonon modes in the Raman spectrum, because of the loss of inversion symmetry. In addition, while the $\mathrm{TO}_{2}$ mode stays hard at $171 \mathrm{~cm}^{-1}$, the low-energy soft $\mathrm{TO}_{1}$ mode displays a distinctive hardening in the ferroelectric state. As seen in the figure, all these features are present, not only in the insulating sample as reported previously ${ }^{14}$, but also in a dilute metallic sample. This implies that the low-temperature optical phonon spectrum of the metallic samples does not differ from their insulating ferroelectric counterparts. Note that the anomalies caused by the ferroelectric transition are identical in the metallic and the insulating samples. This means that the presence of mobile electrons in the solid has no influence on the way the free energy is affected by percolation of electric dipoles. Figure $2 \mathrm{e}$ shows quantum oscillations of resistivity in dilute $\mathrm{Sr}_{1-x} \mathrm{Ca}_{x} \mathrm{TiO}_{3-\delta}$. The frequency of the oscillations does not differ from that measured on Ca-free samples at the same carrier concentration (see Supplementary Information), implying that the presence of the ferroelectric-like order neither hinders the connectivity of the Fermi sea, nor modifies its depth.

Our result puts calcium-substituted-oxygen-reduced strontium titanate in the company of a handful of solids known as 'ferroelectric metals ${ }^{22,23}$. The expression is used to designate a solid in which 
$\mathrm{Sr}_{1-x} \mathrm{Ca}_{x} \mathrm{TiO}_{3-\delta}$
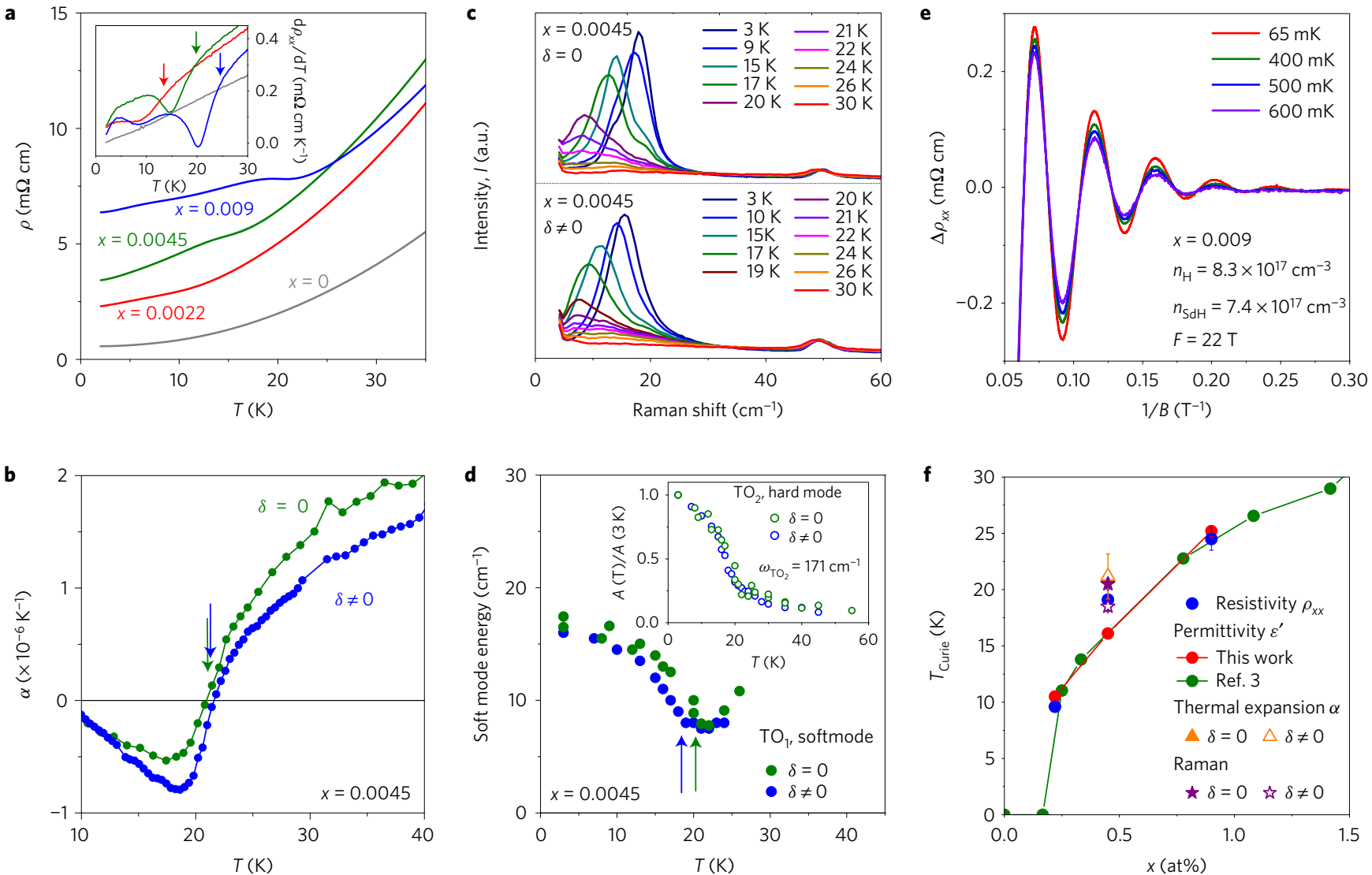

Figure 2 | Coexistence of ferroelectricity with dilute metallicity. a, Temperature dependence of resistivity in metallic $\mathrm{Sr}_{1-x} \mathrm{Ca}_{x} \mathrm{TiO}_{3-\delta}\left(n \sim 7 \times 10^{17} \mathrm{~cm}{ }^{-3}\right)$. Close to the Curie temperature, there is an anomaly, marked by the arrows in the inset showing the temperature dependence of the derivative. $\mathbf{b}$, Thermal expansion in an insulating and a metallic $\left(n=6.6 \times 10^{17} \mathrm{~cm}^{-3}\right)$ sample. Both show identical anomalies at the same temperature. $\mathbf{c}$, Raman spectrum in (insulating) $\mathrm{Sr}_{1-x} \mathrm{Ca}_{x} \mathrm{TiO}_{3}$ and (metallic) $\mathrm{Sr}_{1-x} \mathrm{Ca}_{x} \mathrm{TiO}_{3-\delta}\left(n=9.2 \times 10^{17} \mathrm{~cm}^{-3}\right)$. In both cases, the $\mathrm{TO}_{1}$ soft mode hardens and grows in intensity upon the entry of the system in the ferroelectric state. $\mathbf{d}$, The hardening of $\mathrm{TO}_{1}$ and the change in the integrated intensity of $\mathrm{TO}_{2}$ (inset) triggered by the ferroelectric ordering in the two samples. e, Quantum oscillations in metallic $\mathrm{Sr}_{1-x} \mathrm{Ca}_{x} \mathrm{TiO}_{3-\delta}$. f, Variation of Curie temperature with Ca content according to different experimental probes.

mobile electrons are present when a phase transition structurally indistinguishable from a ferroelectric transition occurs. These systems do not show bulk reversible polarization, the most strict requirement for ferroelectricity. Local probes may document in the future how a Fermi sea coexists with a ferroelectric-like transition. Our bulk probes find that: the presence of the Fermi sea does not attenuate the magnitude of the anomalies caused by the ferroelectric-like transition; and the latter transition does not modify the carrier density of the interconnected Fermi sea. These two features imply that metallicity and ferroelectricity are not confined to different fractions of the sample. Moreover, the two states interact with each other in significant ways, as documented below.

The second result of this study, a quantum phase transition destroying the ferroelectric-like transition, is illustrated in Fig. 3. Figure $3 \mathrm{a}$ shows the temperature dependence of resistivity in $\mathrm{Sr}_{0.991} \mathrm{Ca}_{0.009} \mathrm{TiO}_{3-\delta}$. Resistivity shows both a hump at the ferroelectric transition and a drop at the superconducting transition. The samples become more conducting with increasing $\delta$. Zooming on the data in Fig. $3 b$, one can see three distinct regimes. At low doping, the percolative ferroelectric phase transition introduces a small but clear anomaly in resistivity. Upon further doping, the resistivity displays an upturn (Fig. 3b). Finally, above a critical doping, no anomaly in resistivity is detectable and resistivity recovers a purely quadratic temperature dependence, as seen in $\mathrm{n}$-doped $\mathrm{SrTiO}_{3}$ (refs 24,25). Figure 3c shows the temperature of the resistive anomaly as a function of electron concentration for two different $\mathrm{Ca}$ contents. The ferroelectric instability is destroyed at $5.8 \times 10^{18}$ and $1.7 \times 10^{19} \mathrm{~cm}^{-3}$ for $x=0.0022$ and $x=0.009$, respectively.

What destroys the ferroelectric-like order above a threshold concentration of mobile carriers? We can think of three possibilities. The first possibility is the scenario suggested ${ }^{26}$ in the case of $\mathrm{BaTiO}_{3-\delta}$ (ref. 23). In this scenario, as soon as the Thomas-Fermi screening length, $r_{\mathrm{TF}}=\sqrt{\pi a_{\mathrm{B}}^{*} / 4 k_{\mathrm{F}}}$, becomes shorter than the interdipolar distance, $\ell_{\mathrm{dd}}$ ), the dipole-dipole interaction is screened by mobile electrons and their alignment no longer takes place. However, in the case of $\mathrm{Sr}_{1-x} \mathrm{Ca}_{x} \mathrm{TiO}_{3-\delta}$, as seen in Fig. 3d, the ferroelectric-like order is destroyed when $r_{\mathrm{TF}}$ is still an order of magnitude longer than $\ell_{\mathrm{dd}}$. An intriguing observation points to a second possibility. In all three cases $\left(\mathrm{Sr}_{0.991} \mathrm{Ca}_{0.009} \mathrm{TiO}_{3-\delta}\right.$, $\mathrm{Sr}_{0.998} \mathrm{Ca}_{0.002} \mathrm{TiO}_{3-\delta}$ and $\mathrm{BaTiO}_{3-\delta}$ ), the ferroelectric-like order is destroyed when the Fermi wavevector, $k_{\mathrm{F}}$, is such that $\cos \left(2 k_{\mathrm{F}} \ell_{\mathrm{dd}}\right) \simeq-1$ (see Table 1 and Fig. $3 \mathrm{~d}$ ). A charged impurity inside a Fermi sea is known to generate Friedel oscillations. Interference between oscillations produced by neighbouring impurities is detectable by scanning tunnelling microscopy $(\mathrm{STM})^{27}$. Now, a calcium ion rattling inside its cage in a Fermi sea would generate two superimposed sets of Friedel oscillations, as illustrated in Fig. 3e. Oscillations produced by neighbouring dipoles interfere destructively when $\cos \left(2 k_{\mathrm{F}} \ell_{\mathrm{dd}}\right)=-1$. In addition to these two possibilities, one can think of a purely structural scenario 


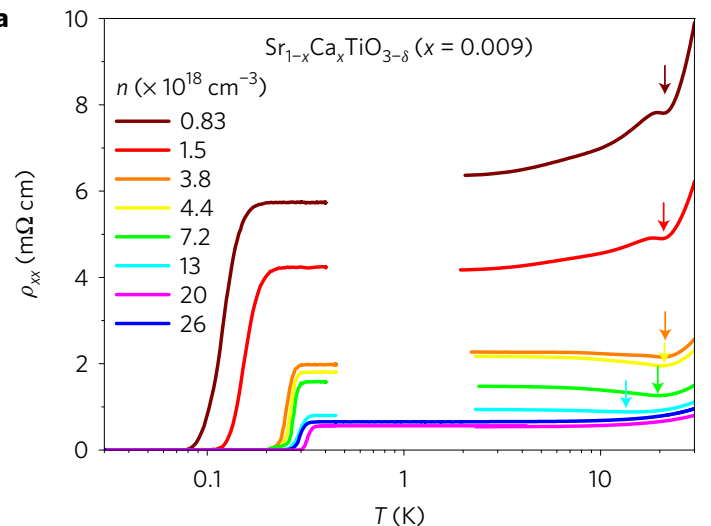

d

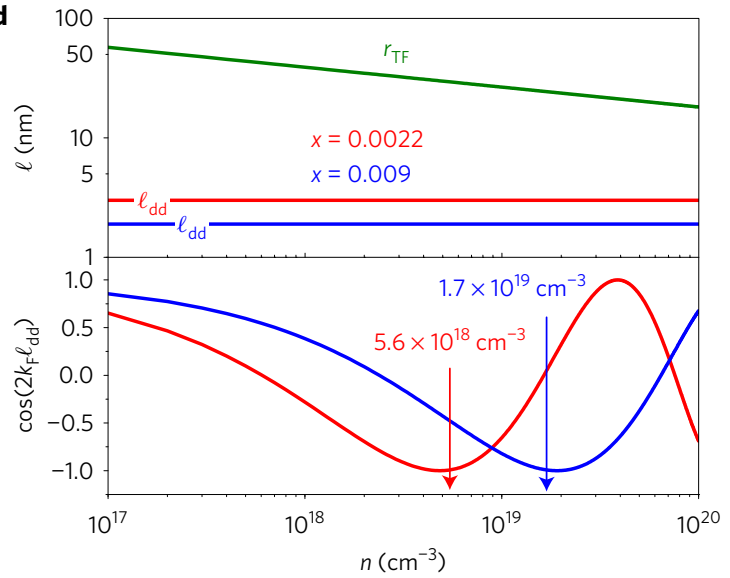

b

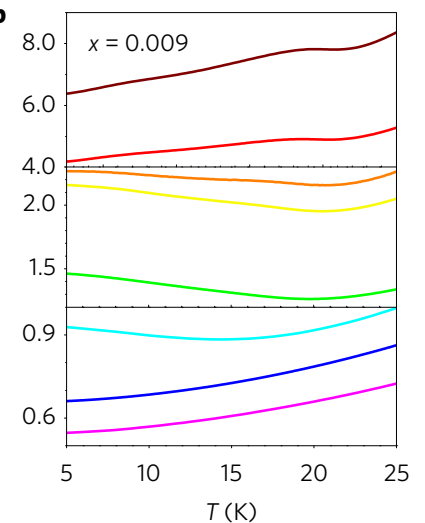

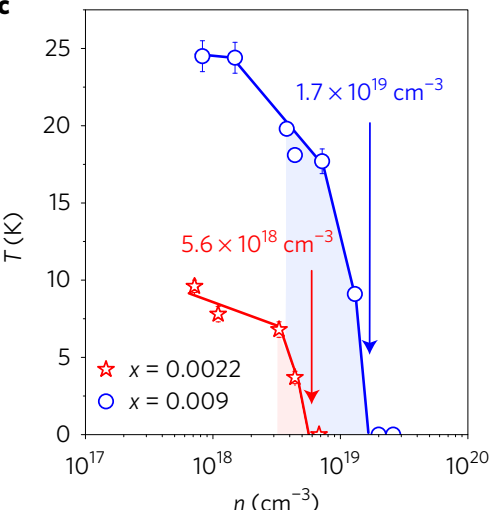

e

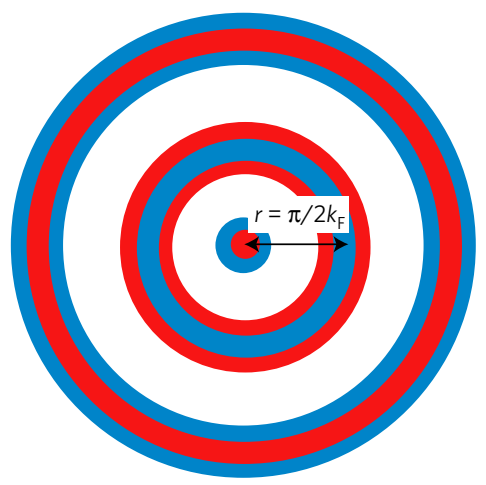

Figure 3 | Evolution of resistivity in $\mathrm{Sr}_{1-x} \mathrm{Ca}_{\mathbf{x}} \mathrm{TiO}_{3-\delta}$ with increasing carrier concentration. a, Temperature dependence of resistivity in $\mathrm{Sr}_{1-x} \mathrm{Ca}_{x} \mathrm{TiO}_{3-\delta}$ $(x=0.009)$ with different carrier concentrations in a semi-logarithmic plot. Note the presence of two phase transitions. The anomaly in the resistivity marked by an arrow is induced by the ferroelectric transition. $\mathbf{b}$, Zoom on the same data, showing the emergence of an upturn in resistivity in the intermediate concentration range and the absence of the ferroelectric anomaly (and the recovery of $T$-square resistivity) at higher concentrations. $\mathbf{c}$, The

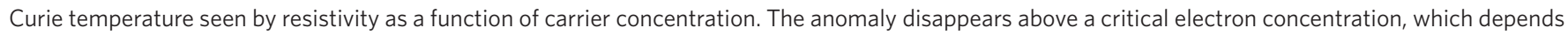

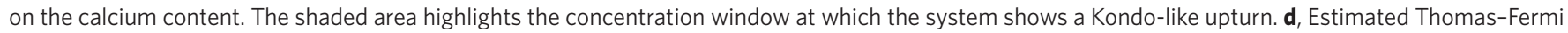
screening length $r_{\mathrm{TF}}$ as a function of carrier density remains much longer than the distance between dipoles, $\ell_{\mathrm{dd}}$. On the other hand, the amplitude of Friedel oscillation is close to -1 , when the ferroelectric order is destroyed for both Ca concentrations. e, Sketch of Friedel oscillations around a dipole in a Fermi sea. The central circle represents a rattling dipole with red and blue referring to opposite polarities. The dipole will generate concentric circles of oscillations minima/maxima, successively in phase and out of phase with the dipole. At a distance $r$ corresponding to $2 k_{F} r=\pi$, the oscillations generated by one dipole interferes destructively with a neighbouring dipole.

Table 1 | A comparison of three solids in which the ferroelectric order is destroyed above a critical density of mobile electrons, $n_{\mathrm{c}}$.

\begin{tabular}{|c|c|c|c|c|c|c|}
\hline Compound & $n_{c}\left(\mathrm{~cm}^{-3}\right)$ & $\ell_{\mathrm{dd}}(\mathrm{nm})$ & $r_{\mathrm{TF}}\left(n=n_{\mathrm{c}}\right)(\mathrm{nm})$ & $k_{\mathrm{F}}\left(n=n_{\mathrm{c}}\right)\left(\mathrm{nm}^{-1}\right)$ & $r_{\mathrm{TF}} / \ell_{\mathrm{dd}}$ & $k_{\mathrm{F}} \ell_{\mathrm{dd}}$ \\
\hline $\mathrm{BaTiO}_{3-\delta}($ refs 23,26) & $1.9 \times 10^{21}$ & 0.4 & 0.4 & 3.82 & 1 & 1.53 \\
\hline $\mathrm{Sr}_{0.991} \mathrm{Ca}_{0.009} \mathrm{TiO}_{3-\delta}$ & $1.7 \times 10^{19}$ & 1.9 & 24 & 0.79 & 12.6 & 1.50 \\
\hline $\mathrm{Sr}_{0.998} \mathrm{Ca}_{0.002} \mathrm{TiO}_{3-\delta}$ & $5.6 \times 10^{18}$ & 3.0 & 29 & 0.55 & 9.7 & 1.65 \\
\hline
\end{tabular}

In all three cases, the dipole alignment is destroyed when the product of the interdipole distance, $\ell_{\mathrm{dd}}$, and the Fermi wave-vector, $k_{\mathrm{F}}$, becomes close to $\pi / 2$. This corresponds to a destructive interference between Friedel oscillations of neighbouring dipoles.

according to which the strain field generated by a calcium site is gradually weakened by the presence of oxygen vacancies. This may impede alignment between sites above a threshold concentration of vacancies. Future research will identify the origin of this quantum phase transition.

Further signature of coupling between dipoles and mobile electrons is the upturn in resistivity at intermediate carrier concentrations (Fig. 3b). The Kondo effect, a many-body resonant scattering due to coupling between the Fermi sea and an alien spin, can give rise to such an upturn. Any quantum degeneracy of a localized state (and not only spin) may produce an Abrikosov-Suhl resonance, and a variety of non-magnetic counterparts of the Kondo effect have been experimentally observed. In PbTe, another dilute metal close to a ferroelectric instability, the introduction of Tl dopants leads to (dilute superconductivity and) an upturn in resistivity, attributed to the charge Kondo effect ${ }^{28}$. A dipolar Kondo effect is a reasonable candidate for explaining the upturn in resistivity seen here. As seen, in Fig. 3c, it occurs only when percolation of the ferroelectric droplets is rapidly degrading with increasing carrier density, providing constraints for any scenario based on Kondo resonance occurring near the quantum phase transition. 


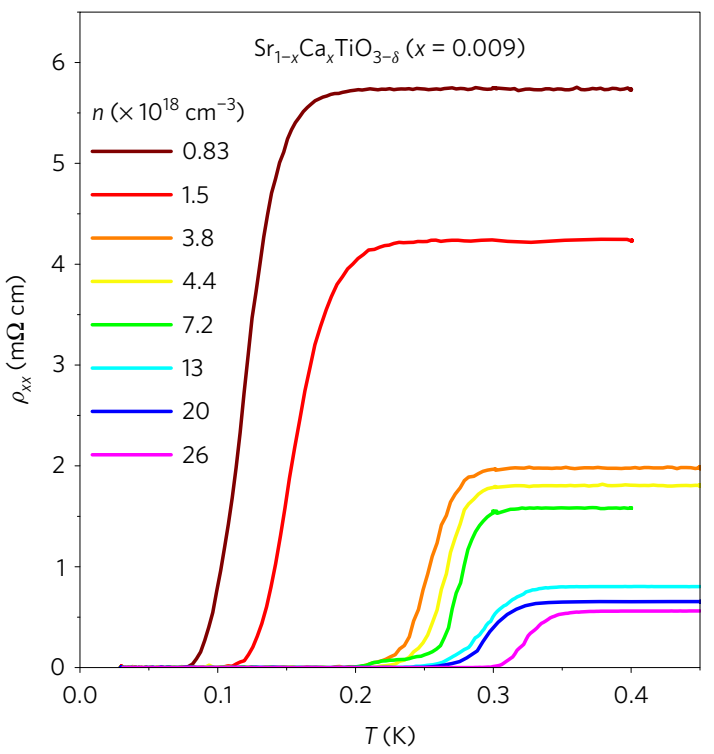

c

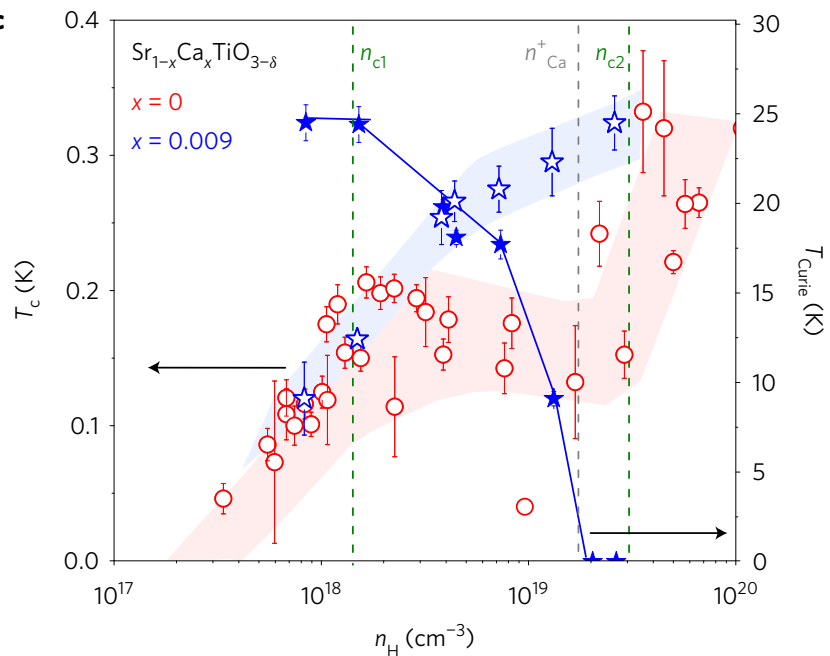

b

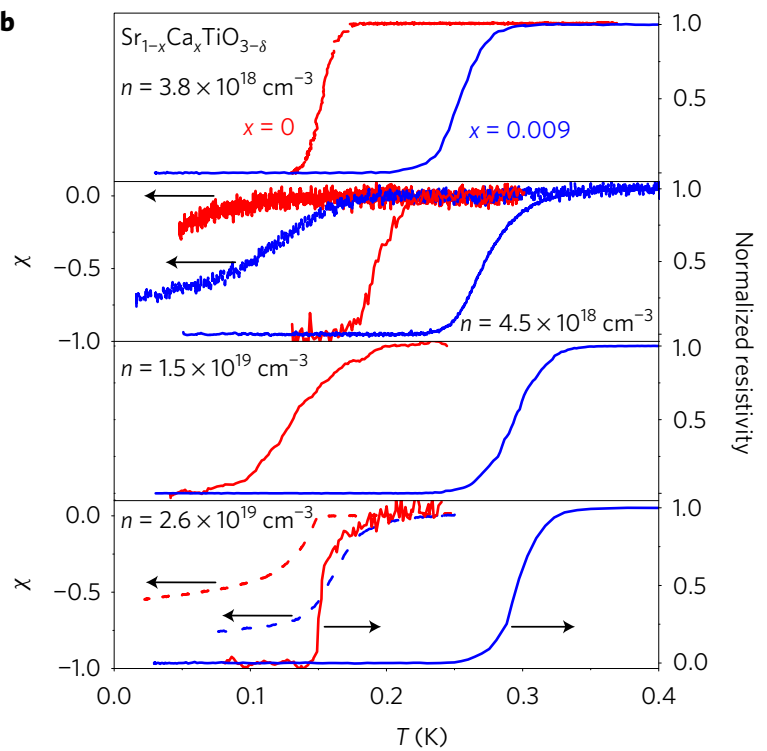

d

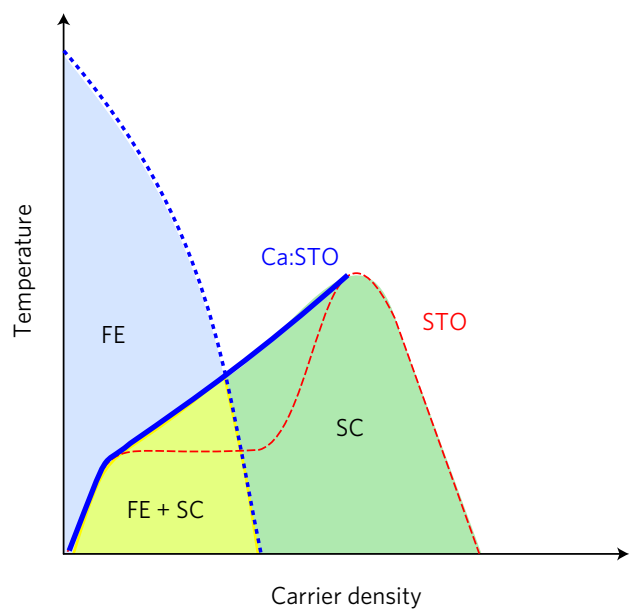

Figure 4 | Evolution of the superconducting transition temperature. a, With increasing doping, the magnitude of normal-state resistivity decreases and the critical temperature rises. b. Superconducting transition seen by resistivity and a.c. susceptibility in Ca-substituted and Ca-free samples with comparable carrier concentration in the range where Ca substitution enhances $T_{\mathrm{c}}$. c. Resistive critical temperature, $T_{\mathrm{c}}$, as a function of carrier concentration in $\mathrm{SrTiO}_{3-\delta}$ and $\mathrm{Sr}_{0.991} \mathrm{Ca}_{0.009} \mathrm{TiO}_{3-\delta}$. Additional Fermi surface pockets emerge at $n_{\mathrm{c} 1}$ and $n_{\mathrm{c} 2}$ (ref. 6). The variation of Curie temperature with carrier concentration is also shown. Note the increase in the superconducting $T_{\mathrm{c}}$ is enhanced in the vicinity of $n_{\mathrm{Ca}}^{*}$, the concentration at which the ferroelectric-like order is destroyed. $\mathbf{d}$, Sketch of the phase diagram showing the region where superconducting (SC) and ferroelectric (FE) order coexist and the increase in $T_{\mathrm{c}}$ induced by Ca substitution.

Figure 4 shows how the superconducting phase diagram is affected by calcium doping in $\mathrm{Sr}_{0.991} \mathrm{Ca}_{0.009} \mathrm{TiO}_{3-\delta}$. As seen in Fig. $4 \mathrm{a}$, with increasing carrier concentration the superconducting transition steadily shifts to higher temperatures. At a given concentration, it occurs at a higher temperature in Ca-substituted samples compared to Ca-free samples (Fig. $4 \mathrm{~b}$ ). Note that the critical temperature according to bulk probes (specific heat, thermal conductivity and magnetic susceptibility) is significantly lower than the onset of the resistive transition ${ }^{29}$. As seen in Fig. 4c, the onset of superconductivity seen by a.c. susceptibility, which sets in below the resistive $T_{\mathrm{c}}$, shifts to a higher temperature after Ca substitution. According to our data, at least in a finite window between the two local maxima close to two critical doping levels, $n_{\mathrm{c} 1}$ and $n_{\mathrm{c} 2}$, of $\mathrm{Ca}$-free $\mathrm{SrTiO}_{3-\delta}$ (ref. 6), superconductivity is strengthened by $\mathrm{Ca}$ substitution. As sketched in Fig. $4 \mathrm{~d}$, there is a region in the phase diagram in which superconductivity and ferroelectricity coexist. Since one cannot separate the orbital and spin components of the order parameter in a non-centrosymmetric superconductor ${ }^{30}$, this region would be a very appealing playground for future research.

The enhancement of critical temperature by $\mathrm{Ca}$ substitution is observed in the vicinity of the quantum phase transition leading to the destruction of ferroelectricity. This provides a new input for the ongoing debate on the microscopic origin of superconductivity ${ }^{4,8-10}$. Calcium substitution leads to a higher dielectric constant at low temperature (Fig. 1b), which would screen Coulomb repulsion between electrons. It hardens the soft ferroelectric mode (Fig. 2b). Both these features may be relevant to the observed enhancement in superconducting $T_{\mathrm{c}}$. Edge et al. ${ }^{9}$ have recently proposed that substituting ${ }^{16} \mathrm{O}$ with ${ }^{18} \mathrm{O}$ should drastically change the superconducting dome of $\mathrm{n}$-doped $\mathrm{SrTiO}_{3}$. They predicted that such a substitution would enhance the highest critical temperature and shift it to lower doping. This is a quantum critical ferroelectric ${ }^{4}$ scenario, in which the maximum $T_{\mathrm{c}}$ is pinned to the destruction of the ferroelectric order. Our observation is in 
qualitative agreement with this scenario. We find that $T_{c}$ is enhanced in the vicinity of the critical doping at which the ferroelectric-like order is destroyed $\left(n_{\mathrm{Ca}}^{*}=1.7 \times 10^{19} \mathrm{~cm}^{-3}\right.$ in $\left.\mathrm{Sr}_{0.991} \mathrm{Ca}_{0.009} \mathrm{TiO}_{3-\delta}\right)$. For this level of Ca content, $n_{\mathrm{Ca}}^{*}$ lies between the two critical doping levels, $n_{\mathrm{c} 1}$ and $n_{\mathrm{c} 2}$, of $\mathrm{Ca}$-free $\mathrm{SrTiO}_{3-\delta}$ where the $T_{\mathrm{c}}$ is almost flat ${ }^{6}$. A definite confirmation of the quantum critical scenario requires an extensive study employing bulk probes ${ }^{29}$ and confirming that the enhancement in critical temperature occurs always near $n_{\mathrm{Ca}}^{*}$, which shifts with Ca content, and is not pinned to $n_{\mathrm{c} 1}$ and $n_{\mathrm{c} 2}$.

\section{Methods}

Methods, including statements of data availability and any associated accession codes and references, are available in the online version of this paper.

\section{Received 20 October 2016; accepted 7 March 2017;} published online 17 April 2017

\section{References}

1. Müller, K. A. \& Burkhard, H. $\mathrm{SrTiO}_{3}$ : an intrinsic quantum paraelectric below 4 K. Phys. Rev. B 19, 3593-3602 (1979).

2. Schooley, J. F., Hosler, W. R. \& Cohen, M. L. Superconductivity in semiconducting $\mathrm{SrTiO}_{3}$. Phys. Rev. Lett. 12, 474-477 (1964).

3. Bednorz, J. G. \& Müller, K. A. $\mathrm{Sr}_{1-x} \mathrm{Ca}_{x} \mathrm{TiO}_{3}$ : an XY quantum ferroelectric with transition to randomness. Phys. Rev. Lett. 52, 2289-2292 (1984).

4. Rowley, S. E. et al. Ferroelectric quantum criticality. Nat. Phys. 10, 367-372 (2014)

5. Lin, X., Zhu, Z., Fauqué, B. \& Behnia, K. Fermi surface of the most dilute superconductor. Phys. Rev. X 3, 021002 (2013).

6. Lin, X. et al. Critical doping for the onset of a two-band superconducting ground state in $\mathrm{SrTiO}_{3-\delta}$. Phys. Rev. Lett. 112, 207002 (2014).

7. Takada, Y. Theory of superconductivity in polar semiconductors and its application to n-type semiconducting $\mathrm{SrTiO}_{3}$. J. Phys. Soc. Jpn 45, 786-794 (1978)

8. Ruhman, J. \& Lee, P. A. Superconductivity at very low density: the case of strontium titanate. Phys. Rev. B 94, 224515 (2016).

9. Edge, J. M., Kedem, Y., Aschauer, U., Spaldin, N. A. \& Balatsky, A. V. Quantum critical origin of the superconducting dome in $\mathrm{SrTiO}_{3}$. Phys. Rev. Lett. 115, 247002 (2015).

10. Gorkov, L. P. Phonon mechanism in the most dilute superconductor: n-type $\mathrm{SrTiO}_{3}$. Proc. Natl Acad. Sci. USA 113, 4646-4651 (2016).

11. Itoh, M. et al. Ferroelectricity induced by oxygen isotope exchange in strontium titanate perovskite. Phys. Rev. Lett. 82, 3540-3543 (1999).

12. Uwe, H. \& Sakudo, T. Stress-induced ferroelectricity and soft phonon modes in $\mathrm{SrTiO}_{3}$. Phys. Rev. B 13, 271-286 (1976)

13. Kleemann, W., Schäfer, F. J., Müller, K. A. \& Bednorz, J. G. Domain state properties of the random-field xy-model system $\mathrm{Sr}_{1-x} \mathrm{Ca}_{x} \mathrm{TiO}_{3}$. Ferroelectrics 80, 297-300 (1988).

14. Kleemann, W., Albertini, A., Kuss, M. \& Lindner, R. Optical detection of symmetry breaking on a nanoscale in $\mathrm{SrTiO}_{3}$ :Ca. Ferroelectrics 203 , 57-74 (1997).

15. Hemberger, J. et al. Quantum paraelectric and induced ferroelectric states in $\mathrm{SrTiO}_{3}$. J. Phys. Condens. Matter 8, 4673-4690 (1996).

16. Carpenter, M. A., Howard, C. J., Knight, K. S. \& Zhang, Z. Structural relationships and a phase diagram for $(\mathrm{Ca}, \mathrm{Sr}) \mathrm{TiO}_{3}$ perovskites. J. Phys. Condens. Matter 18, 10725-10749 (2006).
17. Wang, Y. G., Kleemann, W., Zhong, W. L. \& Zhang, L. Impurity-induced phase transition in quantum paraelectrics. Phys. Rev. B 57, 13343-13346 (1998).

18. Spinelli, A., Torija, M. A., Liu, C., Jan, C. \& Leighton, C. Electronic transport in doped $\mathrm{SrTiO}_{3}$ : conduction mechanisms and potential applications. Phys. Rev. B 81, 155110 (2010)

19. Edwards, P. P. \& Sienko, M. J. Universality aspects of the metal-nonmetal transition in condensed media. Phys. Rev. B 17, 2575-2581 (1978).

20. Behnia, K. On mobility of electrons in a shallow Fermi sea over a rough seafloor. J. Phys. Condens. Matter 27, 375501 (2015).

21. Allen, S. J. et al. Conduction-band edge and Shubnikov-de Haas effect in low-electron-density $\mathrm{SrTiO}_{3}$. Phys. Rev. B 88, 045114 (2013).

22. Shi, Y. et al. A ferroelectric-like structural transition in a metal. Nat. Mater. 12 1024-1027 (2013).

23. Kolodiazhnyi, T., Tachibana, M., Kawaji, H., Hwang, J. \& Takayama-Muromachi, E. Persistence of Ferroelectricity in $\mathrm{BaTiO}_{3}$ through the insulator-metal transition. Phys. Rev. Lett. 104, 147602 (2010).

24. Lin, X., Fauqué, B. \& Behnia, K. $T^{2}$ resistivity in in a small single-component Fermi surface. Science 349, 945-948 (2015).

25. van der Marel, D., van Mechelen, J. L. M. \& Mazin, I. I. Common Fermi-liquid origin of $T^{2}$ resistivity and superconductivity in n-type $\mathrm{SrTiO}_{3}$. Phys. Rev. B 84, 205111 (2011)

26. Wang, Y., Liu, X., Burton, J. D., Jaswal, S. S. \& Tsymbal, E. Y. Ferroelectric instability under screened Coulomb interactions. Phys. Rev. Lett. 109, 247601 (2012)

27. Petersen, L. et al. Direct imaging of the two-dimensional Fermi contour: Fourier-transform STM. Phys. Rev. B 57, R6858-R6861 (1998).

28. Matsushita, Y., Bluhm, H., Geballe, T. H. \& Fisher, I. R. Evidence for charge Kondo effect in superconducting Tl-doped PbTe. Phys. Rev. Lett. 94, 157002 (2005)

29. Lin, X. et al. Multiple nodeless superconducting gaps in optimally doped $\mathrm{SrTi}_{1-x} \mathrm{Nb}_{x} \mathrm{O}_{3}$. Phys. Rev. B 90, 140508(R) (2014).

30. Bauer, E. \& Sigrist, M. (eds) Non-Centrosymmetric Superconductors, Introduction and Overview (Springer, 2012).

\section{Acknowledgements}

This work has been supported by ANR (through the SUPERFIELD and QUANTUM LIMIT projects), by an Ile de France regional grant, by Fonds ESPCI-Paris, by DFG research grant HE-3219/2-1 and by the Institutional Strategy of the University of Cologne within the German Excellence Initiative. X.L. is supported by the Alexander von Humboldt Foundation.

\section{Author contributions}

K.B. conceived the project. C.W.R. and X.L. prepared oxygen-deficient samples and performed resistivity measurements with B.F.; J.H. designed the electric permittivity set-up and carried out the measurements with X.L., C.P.G., D.F. and S.H.; J.H., C.P.G. and D.F. measured sound velocity. T.L. and J.E. performed thermal expansion measurements. Y.G. performed Raman scattering measurements. K.B., C.W.R., X.L. and J.H. wrote the paper. All authors discussed the results and contributed to the manuscript.

\section{Additional information}

Supplementary information is available in the online version of the paper. Reprints and permissions information is available online at www.nature.com/reprints. Publisher's note: Springer Nature remains neutral with regard to jurisdictional claims in published maps and institutional affiliations. Correspondence and requests for materials should be addressed to K.B.

\section{Competing financial interests}

The authors declare no competing financial interests. 


\section{Methods}

Commercial $\mathrm{SrTiO}_{3}$ and $\mathrm{Sr}_{1-x} \mathrm{Ca}_{x} \mathrm{TiO}_{3}(x=0.0022,0.0045$ and 0.009$)$ single crystals were used for this study. The nominal calcium concentration of two samples was checked using the secondary ion mass spectrometry (SIMS) analysis technique as detailed previously ${ }^{31}$. The oxygen content has been changed by heating the samples in vacuum (pressure $10^{-6}-10^{-7} \mathrm{mbar}$ ) to temperatures of $775-1,100^{\circ} \mathrm{C}$. To attain carrier densities above $\sim 4 \times 10^{18} \mathrm{~cm}^{-3}$, a piece of titanium has been placed next to the sample during heating. Ohmic contacts have been realized prior to oxygen removal by evaporation of gold contact pads.

The electrical measurements have been performed in a Quantum Design Physical Property Measurement System (PPMS) between 1.8 and $300 \mathrm{~K}$ as well as in a $17 \mathrm{~T}$ dilution refrigerator with a base temperature of $26 \mathrm{mK}$. Detailed electrical transport information on all samples presented in the main text is listed in the Supplementary Information.

The a.c. magnetic susceptibility was measured in a home-made set-up, comprising a primary coil and a compensating pick-up coil with two sub-coils with their turns in opposite direction. A lock-in amplifier was utilized to supply the exciting a.c. current and pick up the induced voltage signal. The applied a.c. field was as low as $10 \mathrm{mG}$ with a frequency of $16 \mathrm{kHz}$.

The dielectric permittivity measurements were performed employing a frequency-response analyser (NOvOCONTROL Alpha-Analyzer). Using silver paint, the plate-like samples were prepared as capacitors with typical electrode dimensions of $3 \times 3 \mathrm{~mm}^{2}$ and a typical thickness of $0.5-0.85 \mathrm{~mm}$. For the evaluation of the as-measured data $C_{\text {meas }}$, passivated surface layers were assumed, as described in ref. 3. Such layers can be considered as additional capacitors $C_{\text {surf }}$ in series to the remaining bulk specimen $C_{\text {bulk }}$, which limits the total capacitance data. Therefore the data were corrected assuming a temperature-independent surface contribution $C_{\text {bulk }}-1=C_{\text {meas }}-1-C_{\text {surf }}-1$, which results in $\varepsilon(T)$ curves comparable to literature data on surface-etched samples ${ }^{3}$. Measurements of $P(E)$-hysteresis loops were performed using the same set-up with an additional high-voltage module (NOvOCONTROL HVB1000). The actual field-dependent polarization was calculated from the nonlinear dielectric permittivities up to the tenth order, as described in ref. 32 . The thermo-remanent polarization data were gained from the integrated pyro-current as collected with an electrometer (Keithley 6517) after cooling in a poling field of approximately $120 \mathrm{~V} \mathrm{~mm}^{-1}$.

A home-built capacitance dilatometer has been used to detect the uniaxial length changes $\Delta L(T)$ while continuously heating the crystal from about 5 to $150 \mathrm{~K}$ at a rate of about $0.1 \mathrm{~K} \mathrm{~min}^{-1}$. Here, $\Delta L(T)$ was measured along the [100] directions of $\mathrm{Sr}_{1-x} \mathrm{Ca}_{x} \mathrm{TiO}_{3-\delta}$ single crystals with total lengths $L_{0} \simeq 2 \mathrm{~mm}$ and the uniaxial thermal expansion coefficient $\alpha=\left(1 / L_{0}\right)(\partial \Delta L / \partial T)$ has been derived numerically.

The Raman measurements were performed using the $532 \mathrm{~nm}$ line of a diode-pumped solid state (DPSS) laser. An incident power of $5 \mathrm{~mW}$ was focused on a spot of approximate dimensions $50 \times 80 \mu \mathrm{m}$. Power dependence measurements at low temperature indicated negligible laser heating for this incident power. The inelastically scattered photons were analysed using a triple grating spectrometer working in subtractive configuration and equipped with a nitrogen-cooled charge coupled device (CCD) camera. The spectral resolution was about $1.5 \mathrm{~cm}^{-1}$. All spectra were recorded with linearly polarized and parallel incoming and outgoing photons. The crystals were cooled using a close-cycle optical cryostat with a base temperature of $3 \mathrm{~K}$.

Data availability. The data that support the plots within this paper and other findings of this study are available from the corresponding author upon reasonable request.

\section{References}

31. de Lima, B. S. et al. Interplay between antiferrodistortive, ferroelectric, and superconducting instabilities in $\mathrm{Sr}_{1-x} \mathrm{Ca}_{x} \mathrm{TiO}_{3-\delta}$. Phys. Rev. B 91, 045108 (2015).

32. Niermann, D. et al. Domain dynamics in the multiferroic phase of $\mathrm{MnWO}_{4}$. Phys. Rev. B 89, 134412 (2014) 